\title{
CHANGING HYBRID ORGANIZATIONS BY DESIGN
}

\author{
JANINA KLEIN \\ Vrije Universiteit Amsterdam, School of Business and Economics \\ De Boelelaan 1105, 1081 HV Amsterdam, The Netherlands \\ JOHN M. AMIS \\ University of Edinburgh
}

\section{INTRODUCTION}

There is continuing interest in how hybrids, organizations that instantiate values and practices stemming from multiple institutional logics (Battilana et al., 2017; Fossestol et al., 2015), can maintain their hybridity and enact opposing goals sustainably (Battilana, 2018; Smith $\&$ Besharov, 2019). Past research has identified two design approaches that hybrids usually apply: differentiation and integration (Battilana et al., 2017; Smith \& Besharov, 2019).

Differentiation involves structurally separating conflicting elements while integration involves bringing the conflicting elements together (Lawrence \& Lorsch, 1967; Battilana \& Lee, 2014). Research has provided examples of organizations applying either a differentiation (Battilana et al., 2012; Battilana et al., 2015) or integration (Battilana \& Dorado, 2010; Ebrahim et al., 2014; Mair et al., 2015; Pache \& Santos, 2013) approach and, recently, work has started to explore a combination of both (Smets et al., 2015; Smith \& Besharov, 2019).

However, the research in this area to date has demonstrated that while particular design options can help organizations in sustaining their hybridity in some cases, at other times they lead to severe disruption and failure. Thus, the question of how hybrid organizations can be most effectively designed remains unresolved, leading to calls for more systematic investigations into the outcomes of different hybrid designs (Battilana \& Lee, 2014; Smith \& Besharov, 2019). Such work is essential if we are to advance our understanding about how hybrid organizations can sustain their enactment of multiple institutional logics. This paper addresses this gap in our understanding and develops theoretical insights into how and when differentiation and integration influence the sustainability of an organization's hybridity.

\section{ORGANIZATIONAL DESIGN APPROACHES IN HYBRID ORGANIZATIONS}

Galbraith (1977: 5) seminally defined organizational design as "the search for coherence between strategy (domain, objectives and goals), the mode of organization (division into subtasks, coordination to perform the totality of the tasks), and integration of people (selection and training of personnel and design of a rewards system)". As Greenwood and Miller (2010: 79) noted, "[d]esign drives the way strategies are formulated or formed, and determines whether and how they can be implemented. It is the vehicle by which firms recognize the need for adaptation, determine its course, and put change into effect." Organizational design, therefore, plays a crucial role in organizations and seems to be of particular importance in hybrid organizations, which "pursue multiple objectives at once" (Battilana et al., 2015: 1680).

Hybrid organizations instantiate values and practices stemming from multiple, potentially incompatible, institutional logics (Battilana et al., 2017; Fossestol et al., 2015; Smets et al., 2015). Institutional logics constitute the taken-for-granted beliefs and practices that influence 
actors' behaviour and prescribe what is appropriate in a given institutional field (Friedland \& Alford, 1991; Thornton et al., 2012). Through their embeddedness in multiple institutional logics, hybrid organizations experience institutional complexity, a situation in which conflicting institutional logics prescribe divergent and incompatible "rules of the game" (Besharov, 2014; Greenwood et al., 2011), leading hybrids to "deviate from socially legitimate templates for organizing, and thus experience[ing] unique organizing challenges" (Battilana \& Lee, 2014: 397). Scholars have uncovered two approaches to organizing hybridity: integration and differentiation. "Through integration, hybrids amalgamate the different components they bring together, creating a unified blend. In contrast, through differentiation, hybrids keep their constituent elements separate, forming an organization with multiple, distinct parts" (Battilana et al., 2017). Recent work (e.g., Smets et al., 2015; Smith \& Besharov, 2019) has shown that organizations can also apply strategies that combine both approaches. We do not know, however, under which circumstances the various design strategies are more or less suitable.

Besharov and Smith (2014) developed a framework of how multiple institutional logics can influence hybrid organizations along two dimensions: the degree of logic compatibility, "the extent to which the instantiations of logics imply consistent and reinforcing organizational actions" (Besharov \& Smith, 2014: 367) and the degree of logic centrality, "the degree to which multiple logics are treated as equally valid and relevant to organizational functioning" (Besharov \& Smith, 2014: 369). This leads to four ideal-type combinations: contested (logic centrality is high, compatibility is low), aligned (logic centrality is high, compatibility is high), estranged (logic centrality is low, compatibility is low) and dominant (logic centrality is low, compatibility is high). These different types result in different levels of conflict inside the organization with the contested type experiencing extensive, the estranged type moderate, the aligned type minimal and the dominant type experiencing no conflict. The authors do not offer any insights, however, on how organizational design influences internal conflict. The variation into the organizational outcomes of differentiation and integration (Battilana, 2018; Battilana et al., 2017; Smith \& Besharov, 2019) has therefore resulted in the question of how hybrid organizations can be most effectively designed remaining unresolved.

\section{METHODS}

Our research is focused on a national charity, Pathfinder (a pseudonym), that is engaged in preserving and providing public access to some of its country's most significant natural and physical history. Specifically, we concentrate on the design and implementation of a major transformation program intended to address a major financial crisis that threatened its existence. We drew on three sources of data: interviews, observation and documents. We analysed more than one hundred documents such as founding articles, minutes of meetings, annual reports, financial statements, corporate reviews, internal surveys, team briefings, marketing and induction materials, change communications, and strategic and corporate plans. Newspaper articles and books complemented these documents. In addition to the document analysis, we conducted 58 semi-structured interviews across four stakeholder groups: employees (senior managers, middle managers and non-executive staff), board members, members and volunteers. We also spent a total of 86 hours engaged in observation and participant-observation.

First, drawing on our data collected from documents and interviews, we were able to establish the institutional logics that influenced Pathfinder. Following protocols established elsewhere (Dunn \& Jones, 2010; Smets et al., 2012; Toubiana \& Zietsma, 2017) we inductively 
uncovered two logics (Reay \& Jones, 2016). Second, we uncovered the structural arrangements within Pathfinder before and after the change program using a grounded theory approach to data analysis (Gioia et al., 2013). This approach is based on an interpretive method applied to a single case. While collecting data, we travelled back and forth between the literature and emerging themes from our data (Miles \& Huberman, 1994). As such, data were collected and analysed until we reached a point of theoretical saturation (Glaser \& Strauss, 1967).

\section{FINDINGS}

As a preservation charity that at the same time needs to generate income, we found that Pathfinder is influenced by multiple institutional logics that result in competing demands. We term these preservation and business logics, a full description of which can be found in the full version of the paper.

Prior to the change program, Pathfinder operated in what Besharov and Smith (2014) refer to as an estranged type. Logic centrality was low as the preservation logic guided core operations while the business logic played a peripheral role. The preservation and business logics were characterized by low compatibility, characterized by moderate conflict. The main goal of the change program was to, in our terms, bring greater balance to the preservation and business logics. As the change program was completed, Pathfinder developed into a contested type (Besharov \& Smith, 2014) in which both logics were viewed as central, yet provided contradictory prescriptions for action.

Traditionally, Pathfinder was characterized by a differentiated structural arrangement. The organization had a highly centralized design with subunits based on professional groups and regional sites that functioned as separate business units. Preservation specialists were favored in hiring and socialization practices and the organization lacked an integrated performance management system. An overt focus on technical expertise led to the separation of goals, activities, identities and people. Tensions arose between those representing the preservation and the business sides of Pathfinder, particularly over resource allocations. Moreover, because of the overt prioritization of preservation expertise, the organization was mainly staffed with experts in their respective fields rather than individuals with a broader business skillset. Property managers, for example, often lacked the necessary financial skills or desire to run their properties as businesses with many making sustained financial losses.

After the change program, Pathfinder developed an integrated-differentiated structural arrangement in which different approaches were combined. The implementation of a decentralized, regional operating model in which the responsibility for preservation and business performance was assigned to regional teams was at the core of the implemented design changes. The new design grouped properties geographically overseen by a newly created role of regional general manager. The new regional structure, the creation of teams that focused on the customer, the appointment of multi-skilled general and property managers and the introduction of an integrated performance management system that measured preservation and business goals, created a substantively changed design. A differentiated approach was retained at lower hierarchical levels, with separation of preservation and economic specialists. Individuals at the property manager level and higher, on the other hand, were expected to merge preservation and business activities. Hence, while hiring and socialization practices remained differentiated at the lower levels, an integrated approach was applied at the level of property managers and above. The move from a differentiated design to an integrated-differentiated design led to organizational 
outcomes that, in the eyes of many stakeholders we spoke to, helped Pathfinder to overcome the challenges it had faced in the past by becoming commercially more robust while continuing to preserve areas of national significance.

\section{DISCUSSION}

Our findings allow us to contribute to the growing body of literature that has applied an institutional approach to the study of hybrid organizations (e.g., Battilana et al., 2015).

\section{Structural Hybridity Arrangements and the Instantiation of Multiple Institutional Logics}

Building on our empirical insights, we developed four ideal types of structural arrangements in hybrid organizations that can be used to manage multiple institutional logics and organizing practices inside hybrid organizations: fully integrated (integration approach used at all levels), fully differentiated (differentiation approach used at all levels), differentiatedintegrated (differentiation approach used at the upper levels, integration approach used at the lower levels) and integrated-differentiated (integration approach used at the upper levels, differentiation approach used at the lower levels). In the following, we discuss the effectiveness of each configuration in different institutional settings by drawing on Besharov and Smith's (2014) model.

Based on our findings, we argue that the fully integrated approach best fits aligned types that are characterized by high logic centrality and high logic compatibility. In these organizations, logic centrality is high, and logic compatibility is high so that institutional logics prescribe consistent implications for organizational practices in terms of goals, activities and people. Similarly, we argue that the fully integrated approach best fits the dominant type in which logic centrality is low and logic compatibility is high. Similar to the aligned type, in the dominant type, the institutional logics the organization is embedded in place consistent demands on the organization. Since the logic compatibility is high in both types and the logics prescribe consistent hybrid practices, these two types can apply a fully integrated approach and thus leverage the benefits of integration without this causing extensive tensions.

When logic compatibility is low, however, a fully integrated approach cannot be used because "logics offer inconsistent implications for organizational action, leading actors to grapple with divergent goals and divergent means of achieving these goals" (Besharov \& Smith, 2014: 372). Under such circumstances, an integration approach would create extensive tensions. In estranged types, logic centrality is low, which results in one logic exerting a dominant influence on the organization. Based on our findings, we argue that the fully differentiated approach will allow organizations to keep internal conflicts at a minimum.

However, although a fully differentiated approach best fits estranged types in terms of the minimization of internal conflict, it has to be highlighted that this arrangement will further empower the central logic, something that might not be desirable. This is something we observed at Pathfinder before the change program with the preservation logic dominant and the business logic playing only a peripheral role. The different logics were effectively, if unintentionally, restricted to different subunits, activities and practices, thus minimizing internal conflict by structurally separating their contradictory demands. This arrangement, as we explained, reinforced the central role of the preservation logic, something that was perceived to be a reason for Pathfinder's poor financial performance. This develops previous work that has suggested 
differentiation in organizations can decrease the centrality of competing logics (Kraatz \& Block, 2008; Reay \& Hinings, 2009).

The minimization of conflict in contested types has been a focus of recent research, and it has been argued that, in such organizations, a differentiation strategy cannot be used (Gümüsay et al., 2019). Ramus et al. (2017: 1255) argued that this is "because it would cause organizational paralysis and overt power conflicts that could also jeopardize the organization's survival." Gümüsay et al. (2019), building on Besharov and Smith's (2014) model, support this reasoning, suggesting that applying a differentiation strategy in contested hybrids might leave the organization estranged. Consequently, the integration strategy remains the likely strategy for contested hybrids, however, as we demonstrated here, this may not be a viable option because "logics offer inconsistent implications for organizational action, leading actors to grapple with divergent goals and divergent means of achieving these goals" (Besharov \& Smith, 2014: 372). None of the approaches, consequently, has been found to be applicable in the case of contested types. This challenge, we argue, can be overcome by combining approaches, as we now explain.

We show that, rather than only combining different approaches in terms of differentiation and integration across different practices (e.g., an organization can apply an integrated approach in terms of people and a differentiated approach in terms of goals), organizations can also combine different approaches across managerial levels or groups of people. In our case, we found an integrated approach at the upper levels and a differentiated approach lower down. While recent work (Smets et al., 2015; Smith \& Besharov, 2019) has started to explore combinations of differentiation and integration, we extend this work by uncovering how configurations can be systematically and deliberately varied across different practices and managerial levels. Our work thus enhances our understanding of how organizations can combine approaches by uncovering that combinations are not only possible in terms of different practices but also within practices. This is an important insight because people can perceive social and economic values as either complementary or conflicting (Besharov, 2014).

We suggest that an integrated-differentiated approach provides contested hybrid organizations with a means to structure their hybridity in a sustainable way. We thus provide one answer to the question of how contested types, which cannot apply either integration or differentiation approaches (Gümüsay et al., 2019), can constructively engage central and incompatible logics, a question that scholars have recently acknowledged as central to our developing understanding of hybrid organizations (Gümüsay et al., 2019). Thus, "instead of treating the differentiated-integrated distinction as static" (Smith \& Besharov, 2019: 31) we uncover how leaders can change hybridity arrangements.

\section{Managing Institutional Complexity by Organizational Design}

Our work thus provides insights that enable us to reconnect work on organizational design (Gulati \& Puranam, 2009; Lawrence \& Lorsch, 1967; Worley \& Lawler, 2010) with studies on institutional complexity (Greenwood, Hinings, \& Whetten, 2014; Greenwood \& Miller, 2010). Scholars in this area have been interested in organizational responses to institutional complexity and in particular how they manage the tensions that arise from organizations' embeddedness in conflicting institutional logics (Battilana et al., 2015). Previous work has revealed that organizations tend to either "blend" or "structurally separate" logics. We extend this work by explaining how different strategies can be combined depending on the institutional context. This finding is particularly helpful for our understanding of contested 
hybrids that are unable to either blend or structurally separate logics (e.g., Gümüsay et al., 2019; Ramus et al., 2017).

Further, we show that leaders can deliberately change an organization's logic instantiation through design changes and hence can influence how institutional complexity plays out inside hybrid organizations. While previous work has shown that logic instantiations inside organizations can change in response to an external shock (Ramus et al., 2017), and it has been suggested that the instantiations of multiple logics inside organizations might change over time (Besharov \& Smith, 2014), our work is one of the first studies to explicitly examine how organizational leaders reconcile institutional complexity through design changes. This is important since it shifts our attention away from institutional complexity as something that is externally imposed on organizations towards an understanding in which the internal experience of institutional complexity can be strategically altered (Smith \& Besharov, 2019). Our insights, therefore, build on and extend work that has conceived of organizational design as something that organizations do, rather than something they have (Karim et al., 2016; Pasmore et al., 2019) and assigns organizational actors a higher level of agency in institutional complexity.

REFERENCES AVAILABLE FROM THE AUTHOR(S) 